\title{
Finding the optimum times to flip a beefsteak using a genetic algorithm
}

\author{
Rebekah Yu-En Chin* \\ Project advisor: Prof. Leevan Ling ${ }^{\dagger}$
}

Abstract. This paper attempts to find the best times at which to flip a beefsteak so that the steak is cooked to medium-rare, subject to a fixed minimum temperature. The steak, pan, and a layer of oil is modeled with a partial differential equation. The physical parameters of the model are approximated and their effects on the model are discussed. Appropriate boundary conditions are selected to allow for heat convection with the air, heat to enter from a stove, and heat diffusion between the steak, oil, and pan. The model is compared to experimental results and evaluated. The model is then converted into an optimization problem and minimized with a genetic algorithm (GA). The solution obtained with GA lists the optimum times to flip a steak to minimize its mean temperature and performed better than a single-flip procedure.

1. Introduction. In practice, there are two very different approaches to cooking a steak; flipping the steak once in the entire heating process, as in $[7,16]$, and flipping it every minute, as in [20]. The latter approach can be modified so that the interval between flips is reduced to 30 seconds or increased to two minutes, as is done in this paper.

The mathematical modeling of single-sided contact heating of food has been done in $[5,11$, 14] while [2] found that double-sided contact cooking with a variable heat source was superior to constant heat. However, these papers did not consider models that allowed for multiple flips as seen in [20].

In this paper, we attempt to find a sequence of flips, where the time interval can be as short as 30 seconds, using a genetic algorithm with the goal of producing a medium-rare steak. Such a steak should have a mean temperature of between 55 and $65^{\circ}$ Cand a uniform temperature gradient when moving from the inner part of the steak to the outer surfaces. A steak with a mean of about $60{ }^{\circ} \mathrm{C}$ would indicate a high quality steak. Furthermore, the steak must be edible and thus, cannot have points that are below $50{ }^{\circ} \mathrm{C}$.

The main equation governing the heating process of the steak is the heat equation, with boundary conditions to allow convection with the air. Heat entering the system is modeled as a Dirichlet boundary condition while diffusion and convection is modeled as a Neumann boundary condition. Water is also allowed to evaporate from the steak by modeling this process as a heat sink within the steak.

The steak and the pan is modeled and solved numerically with the finite-element method then minimized with the genetic algorithm. The model derived is also verified by comparing the results obtained to empirical results.

As we want the steak to be flipped at most once every $t_{f}$ seconds, this results in a highly discontinuous and non-linear equation. One method to solve such an equation is to use a brute-force method like a genetic algorithm.

Given a function, a genetic algorithm attempts to find the minimum (or maximum) of

\footnotetext{
* Department of Mathematics, Hong Kong Baptist University rebekahchin@life.hkbu.edu.hk

${ }^{\dagger}$ Department of Mathematics, Hong Kong Baptist University lling@hkbu.edu.hk
} 
the function by evaluating a number of randomly-generated solutions [12]. It was inspired by Darwinian evolution in that each solution is evaluated then given a fitness score. Solutions with higher fitness scores then have a higher probability of being selected to undergo "reproduction" by exchanging sub-sequences of their solution to create new solutions. Solutions also randomly "mutate" to introduce new solutions into the population.

During the cooking process, the meat loses water through three phenomenons: evaporation, which occurs at the surface, drip, when the muscle fibers are cut, and capillary action, when the muscle fibers shrink. Most of the water loss is due to capillary action, in particular, when the muscle fibers shrink longitudinally. This occurs when the temperature of the meat is between 60 and $70{ }^{\circ} \mathrm{C}$ [19].

The authors of [19] also discuss the change in texture of meat products when heated, reporting that whole meat remains tough up to $50{ }^{\circ} \mathrm{C}$ then becomes most tender between 50 and $65{ }^{\circ} \mathrm{C}$. However, when heated past $65^{\circ} \mathrm{C}$, the meat begins to fracture, indicating increased toughness.

Therefore, we decided to simulate the heating of a steak to at least $50{ }^{\circ} \mathrm{C}$ with the objective of minimizing the overall mean temperature with a genetic algorithm. The genetic algorithm is set up such that it outputs the mean maximum historical temperature, $u_{h}$, of the steak and prefers steaks with lower mean historical temperatures. Maximum historical temperature, $u_{h}$, at a point refers to the maximum temperature of that point over the entire cooking process.

2. Model derivation. In this section, we state the partial differential equation that is used, which is the two dimensional heat equation. We also introduce some key assumptions about the composition of the steak to estimate its thermal diffusivity, and to simplify the process of mass transfer from water evaporation in the steak. As the heat supplied to the steak is unlikely to be constant everywhere in practice, we add a variable heat source at the base of the pan. Additionally, we allow heat loss to occur in the form of convection at the boundaries. We also investigate the effect of pan material, room temperature, and the initial temperature of the steak on the heating process. Finally, we explain the function used to flip the steak, which swaps the temperature of two points at equal distances from the midpoint depth of the steak.

2.1. The partial differential equation. We begin with a general 3-dimensional heat equation in cylindrical coordinates, from Fourier's Law, in some domain $D$ :

$$
\begin{aligned}
\alpha & {\left[\frac{1}{r}\left(\frac{\delta u(r, \theta, z, t)}{\delta r}+x \frac{\delta^{2} u(r, \theta, z, t)}{\delta r^{2}}\right)+\frac{1}{r^{2}} \frac{\delta^{2} u(r, \theta, z, t)}{\delta \theta^{2}}+\frac{\delta^{2} u(r, \theta, z, t)}{\delta z^{2}}\right]+Q(r, \theta, z, t) } \\
& =\frac{\delta u(r, \theta, z, t)}{\delta t}, \quad(r, \theta, z) \in D, t>0
\end{aligned}
$$

Here, $u$ is temperature in the specified domain while $\alpha$ is thermal diffusivity and is defined as:

$$
\alpha:=\alpha(r, \theta, z)=\frac{k}{c \rho}, \quad(r, \theta, z) \in D
$$

where $k$ is thermal conductivity, $c$ is specific heat capacity, and $\rho$ is density.

In the model, we have a steak, a thin layer of oil, and a pan. Then, we assume that the heat transfer in the steak, oil, and pan is primarily governed by the heat equation in (2.1). 


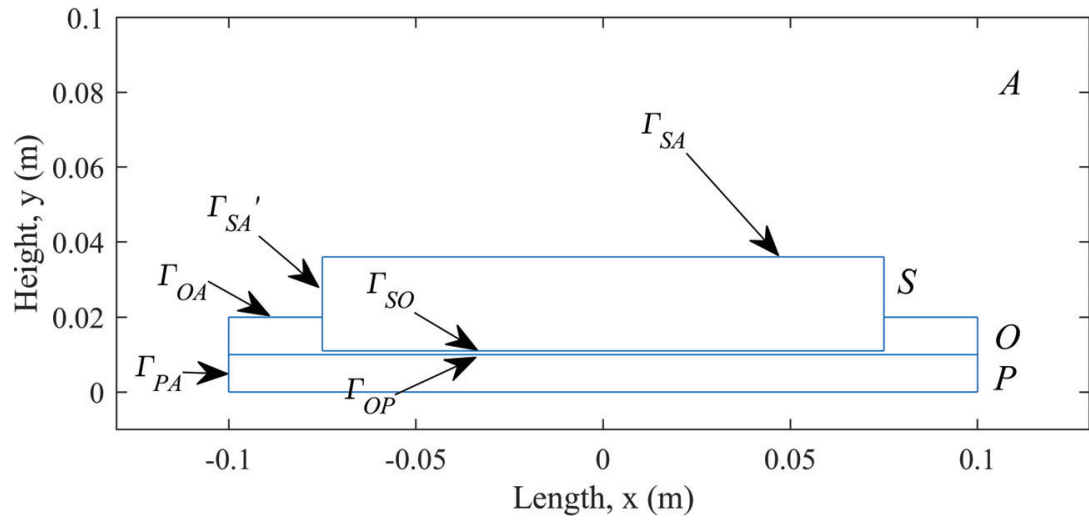

(a) Cross-sectional view of the model.

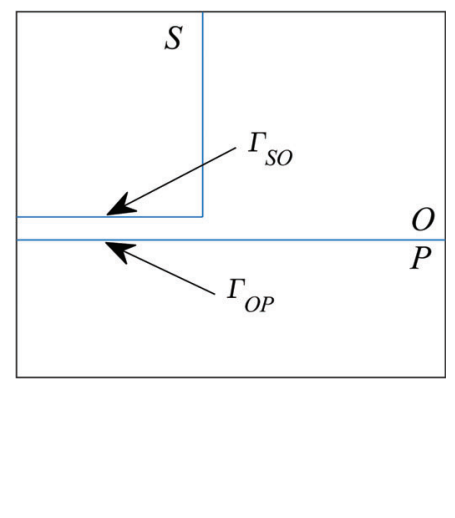

(b) Detail of oil layer.

Figure 2.1: A graphical representation of the model with labeled boundaries.

Additionally, we assume that the steak does not shrink, resulting in a fixed domain throughout the heating process.

Finally, we assume that the model is radially symmetrical to reduce the heat equation from (2.1) to the following 2-dimensional heat equations:

$$
\begin{aligned}
& k_{p}\left[\frac{\delta^{2} u(x, y, t)}{\delta x^{2}}+\frac{\delta^{2} u(x, y, t)}{\delta y^{2}}\right] \quad=c_{p} \rho_{p} \frac{\delta u(x, y, t)}{\delta t}, \quad(x, y) \in P \\
& k_{o}\left[\frac{\delta^{2} u(x, y, t)}{\delta x^{2}}+\frac{\delta^{2} u(x, y, t)}{\delta y^{2}}\right]=c_{o} \rho_{o} \frac{\delta u(x, y, t)}{\delta t}, \quad(x, y) \in O \\
& k_{s}\left[\frac{\delta^{2} u(x, y, t)}{\delta x^{2}}+\frac{\delta^{2} u(x, y, t)}{\delta y^{2}}\right]-Q=c_{s} \rho_{s} \frac{\delta u(x, y, t)}{\delta t}, \quad(x, y) \in S \\
& t>0
\end{aligned}
$$

which are defined on the following domains:

$$
\begin{aligned}
& P=\left\{\left[-x_{p}, x_{p}\right] \times[0, a]\right\}, \\
& O=\left\{O_{1} \cup O_{2} \cup O_{3}\right\}, \\
& S=\left\{\left[-x_{s}, x_{s}\right] \times[a+b, a+b+c]\right\}, \\
& O_{1}=\left\{\left[-x_{p},-x_{s}\right] \times[a, 2 a]\right\}, \\
& O_{2}=\left\{\left[-x_{s}, x_{s}\right] \times[a, a+b]\right\}, \\
& O_{3}=\left\{\left[x_{s}, x_{p}\right] \times[a, 2 a]\right\}, \\
& P, O, S \in \mathbb{R}^{2}
\end{aligned}
$$

where $P, O$, and $S$ is the two dimensional space occupied by the pan, oil, and steak, visualized in Figure 2.1. $u$ is temperature in the specified domain, $t$ is time, and $Q$ is heat loss from mass transfer and is to be defined in subsection 2.4. The parameters $a, b$, and $c$ are the height of the pan, oil layer, and steak respectively.

2.2. A non-constant heat source. In this section, we use a Gaussian function to simulate the temperature of a gas burner with two rings. 
The Gaussian function is as follows:

$$
f(x ; \sigma, \lambda, \mu)=\frac{1}{\sigma \sqrt{2 \pi}} \exp \left(\frac{1}{2} \frac{\mu^{2}(x-\lambda)^{2}}{6^{2}}\right), \quad x \in \mathbb{R}
$$

where $\sigma, \mu$, and $\lambda$ are constants, and $x$ is a variable.

As we want the hottest part of the fire to be $u_{\max }$, the coolest to be at room temperature, $u_{a t m}$, and the area between the two rings to be at most $40{ }^{\circ} \mathrm{C}$ cooler than $u_{\max }$, we use the following equation, $u_{f}$ to simulate the temperature of the fire at the base of the pan:

$$
u_{f}(x)= \begin{cases}f\left(x ; 0.02, \frac{1}{\left(u_{\max }-u_{a t m}\right) \sqrt{2 \pi}}, 200\right), & x \in(-0.044,0.044), \\ f\left(x ; 0.06, \frac{1}{\left(u_{\max }-u_{a t m}\right) \sqrt{2 \pi}}, 300\right), & x \in\left[-x_{p},-0.044\right] \cup\left[0.044, x_{p}\right]\end{cases}
$$

The function in (2.9) results in the heat distribution as seen in Figure 2.2, given the main burner is $2 \mathrm{~cm}$ from the origin while the second burner is in a ring $6 \mathrm{~cm}$ from the origin.

\subsection{Boundary conditions with diffu-} sion and convection. Within the model, we have the diffusion of heat between the pan and the oil, and the oil and the steak. For the heat flux to be continuous between the different domains, we assume that there is perfect thermal contact between the steak, oil, and pan such that the temperature on these shared boundaries are equal. Additionally, on the external faces of the model, we allow for the cooling effect of air by introducing heat transfer by convection.

To achieve this, we assume that only natural, or free convection takes place. In practice, this would translate to not using a kitchen hood when cooking.

According to [8, p.394], heat transfer on

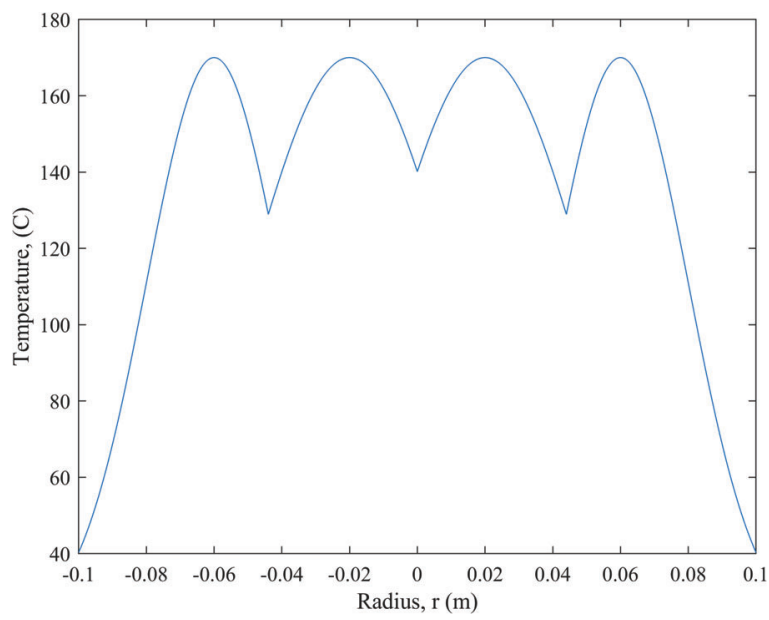

Figure 2.2: Heat distribution at the base of the pan, produced by a gas burner.

$$
k \nabla u+h(u) \cdot u(x, y, t)=h(u) \cdot u_{a t m}, \quad(x, y) \in \delta R,
$$

where $k$ is the thermal conductivity of the solid, $u$ is the temperature of the solid, $h$ is the convection coefficient of air and a function of $u$, and $u_{a t m}$ is the atmospheric temperature.

As the convection coefficient is dependent on the temperature on the boundary ${ }^{1}$, the

\footnotetext{
${ }^{1}$ See Appendix A for the derivation of $h$
} 
boundary conditions of the model are:

$$
\begin{aligned}
u(x, 0, t) & =u_{f}(x), & & (x, y) \in \Gamma_{P A} \\
k_{s} \nabla u(x, y, t)+u(x, y, t) h(u(x, y, t)) & =u_{a t m} h(u(x, y, t)), & & (x, y) \in \Gamma_{S A} \\
k_{s} \nabla u(x, y, t) & =0, & & (x, y) \in \Gamma_{S A}^{\prime} \\
k_{o} \nabla u(x, y, t)+u(x, y, t) h(u(x, y, t)) & =u_{a t m} h(u(x, y, t)), & & (x, y) \in \Gamma_{O A} \\
\left.k_{p} \frac{\delta u(x, y, t)}{\delta y}\right|_{y=a} & =\left.k_{o} \frac{\delta u(x, y, t)}{\delta y}\right|_{y=a}, & & (x, y) \in \Gamma_{O P} \\
\left.k_{O} \frac{\delta u(x, y, t)}{\delta y}\right|_{y=a+b} & =\left.k_{s} \frac{\delta u(x, y, t)}{\delta y}\right|_{y=a+b}, & & (x, y) \in \Gamma_{S O} \\
\left.u_{o}(x, y, t)\right|_{y=a} & =\left.u_{p}(x, y, t)\right|_{y=a}, & & (x, y) \in \Gamma_{O P} \\
\left.u_{s}(x, y, t)\right|_{y=a+b} & =\left.u_{o}(x, y, t)\right|_{y=a+b}, & & (x, y) \in \Gamma_{S O} \\
t & >0 & &
\end{aligned}
$$

where $u_{f}$ is as defined in (2.9), $u$ is temperature in the specified domain while $u_{o}, u_{p}$, and $u_{s}$ are the temperature on the boundary of the domains $O, P$, and $S$ respectively. See Figure 2.1 for a graphical representation of the model and its boundaries.

2.4. Mass transfer in the steak. According to [16], after cooking, the water content of the steaks fell from 75 to about 65 percent while the weight of the steaks decreased by about 30 percent. We theorized that this weight loss is due to water evaporation and fat loss, where fat loss is observed as oil drip when cooking. In this section, we will use mass transfer to account for water loss through evaporation.

From [9, p. 496], we know that the type of boiling that takes place, and the corresponding heat flux, depends on the temperature excess, $\Delta u=u_{w}-u_{s a t}$, where $u_{w}$ is the temperature of the water, and $u_{\text {sat }}$ is the boiling point of water at $1 \mathrm{~atm}$.

Then, using the derivation in Appendix $\mathrm{C}$, the heat flux, $Q$ is:

$$
Q= \begin{cases}\sum_{i} 0.75 \cdot 1000 \cdot 1042(\Delta u)^{1 / 3} \pi\left(\frac{h}{2}\right)^{2}, & \text { when } 100 \leq u_{i} \leq 107.76 \\ \sum_{i} 0.75 \cdot 1000 \cdot 5.56(\Delta u)^{3} 4 \pi\left(\frac{h}{2}\right)^{2}, & \text { when } u_{i}>107.76,\end{cases}
$$

where $\Delta u=u_{i}-100, u_{i}$ is the temperature at the $i^{t h}$ node set by the finite element method, and $h$ is the distance between nodes.

2.5. Initial conditions. We assume that the pan and the oil has been preheated before the steak is added to the system, giving the following initial conditions for the model:

$$
\begin{aligned}
u(x, y, t) & =u_{\max }, & & (x, y) \in P \\
u(x, y, t) & =u_{\text {oil }}, & & (x, y) \in O \\
u(x, y, t) & =u_{\text {ini }}, & & (x, y) \in S \\
t & =0 & &
\end{aligned}
$$


Table 2.1: Physical parameters from subsection 2.6.

\begin{tabular}{|c|c|c|c|}
\hline Symbol & Parameter & Value & Units \\
\hline \multicolumn{4}{|l|}{ Pan } \\
\hline$a$ & Height of pan & 0.01 & $\mathrm{~m}$ \\
\hline$u_{\max }$ & Max temperature of fire & 170 & ${ }^{\circ} \mathrm{C}$ \\
\hline$x_{p}$ & Half-length of pan & 0.01 & $\mathrm{~m}$ \\
\hline$c_{p}$ & Specific heat capacity & 456 & $\mathrm{~J} / \mathrm{kgK}$ \\
\hline$k_{p}$ & Thermal conductivity & 55 & $\mathrm{~W} / \mathrm{mK}$ \\
\hline$\rho_{p}$ & Density & 7920 & $\mathrm{~kg} / \mathrm{m}^{3}$ \\
\hline \multicolumn{4}{|l|}{ Steak } \\
\hline$c$ & Height of steak & 0.025 & $\mathrm{~m}$ \\
\hline$x_{s}$ & Half-length of steak & 0.075 & $\mathrm{~m}$ \\
\hline$u_{\text {ini }}$ & Initial temperature of steak & 20 & ${ }^{\circ} \mathrm{C}$ \\
\hline$u_{\text {atm }}$ & Atmospheric temperature & 20 & ${ }^{\circ} \mathrm{C}$ \\
\hline$c_{m}$ & Specific heat capacity of muscle matrix & 2008 & $\mathrm{~J} / \mathrm{kgK}$ \\
\hline$k_{m}$ & Thermal conductivity of muscle matrix & 0.18 & $\mathrm{~W} / \mathrm{mK}$ \\
\hline$\rho_{m}$ & Density of muscle matrix & 1330 & $\mathrm{~kg} / \mathrm{m}^{3}$ \\
\hline$c_{w}$ & Specific heat capacity of water & 4178 & $\mathrm{~J} / \mathrm{kgK}$ \\
\hline$k_{w}$ & Thermal conductivity of water & 0.57 & $\mathrm{~W} / \mathrm{mK}$ \\
\hline$\rho_{w}$ & Density of water & 997.2 & $\mathrm{~kg} / \mathrm{m}^{3}$ \\
\hline$c_{s}$ & Specific heat capacity of steak & 3635 & $\mathrm{~J} / \mathrm{kgK}$ \\
\hline$k_{s}$ & Thermal conductivity of steak & 0.4725 & $\mathrm{~W} / \mathrm{mK}$ \\
\hline$\rho_{s}$ & Density of steak & 1080.4 & $\mathrm{~kg} / \mathrm{m}^{3}$ \\
\hline \multicolumn{4}{|l|}{ Oil } \\
\hline$b$ & Height of oil layer & 0.002 & $\mathrm{~m}$ \\
\hline$u_{\text {oil }}$ & Initial temperature of oil & 170 & ${ }^{\circ} \mathrm{C}$ \\
\hline$c_{o}$ & Specific heat capacity of oil & 2000 & $\mathrm{~J} / \mathrm{kgK}$ \\
\hline$k_{o}$ & Thermal conductivity of oil & 0.15 & $\mathrm{~W} / \mathrm{mK}$ \\
\hline$\rho_{o}$ & Density of oil & 900 & $\mathrm{~kg} / \mathrm{m}^{3}$ \\
\hline
\end{tabular}

See Table 2.1 for the values of $u_{\max }, u_{o i l}$, and $u_{i n i}$.

2.6. Parameter estimation. As a genetic algorithm minimizes a function with respect to one or more input variables, here, we set the decision variable to be the mean historical temperature of the steak, fixing all other variables besides the time interval between flips, $t_{f}$, before running the algorithm. In particular, we fixed the composition of the steak, the initial temperature of the steak, $u_{i n i}$, and the temperature at the base of the pan, $u_{f}$. We found that other parameters such as oil volume, room temperature, and pan material are less important as they only minimally affect the heating process. We also investigated the effect of different time interval between flips, $t_{f}$, on the heating process. These physical parameters are summarized in Table 2.1.

Composition of the steak. As the thermal diffusivity of a steak depends on the leanness and the cut of the steak, we made several assumptions about the steak in order to obtain a reasonable estimation for this value.

First, we assumed that beefsteak used is lean, with the molecules of water and muscle 
distributed randomly throughout steak, and that 75 percent of the steak consists of water while the remaining 25 percent is muscle. ${ }^{2}$ Also, we assumed that the specific heat capacity, thermal conductivity, and density of the steak remained constant and was a weighted average of water and muscle, where water has a weight of 0.75 , and muscle a weight of 0.25 .

Presence of oil. To improve thermal contact between the steak and the pan and to account for the decrease in oil content after heating, we assumed that a small volume of oil had seeped out of the steak, forming a fixed, $2 \mathrm{~mm}$ layer that completely fills the base of the pan, as seen in Figure 2.1. We further assumed that the oil drip occurs instantaneously upon the addition of the steak to the system.

Room temperature. As seen in subsection 2.3, the convection of heat on the boundary is dependent on the temperature of the surroundings. Since the heat loss from the boundary of the steak increases as ambient temperature decreases, with all other variables held constant, we thought that it would be prudent to investigate the effect of ambient temperature on the time taken for the steak to cook.

As we found that the cooking time to a core temperature of $65{ }^{\circ} \mathrm{C}$ increased by about one minute for every five Celsius decrease in room temperature, the cooking time is minimally affected by the change in room temperature, and we set the room temperature to $20{ }^{\circ} \mathrm{C}$.

Initial temperature of the steak. To determine the relationship between the initial temperature of the steak and the time taken to cook the steak, we tested steaks with an initial temperature of $-2,4$, and $20{ }^{\circ} \mathrm{C}$ or room temperature. We found that frozen steaks took nearly 2 minutes longer to cook to $65^{\circ} \mathrm{C}$ compared to the steaks thawed to room temperature. Thus, we set the initial temperatures of the steaks to $20{ }^{\circ} \mathrm{C}$ to speed up the simulation.

There have been concerns that thawing out a steak to room temperature might be unsafe. However, [1] found that the E. coli population counts in steaks with an initial temperature of $-5,4$, and $24^{\circ} \mathrm{C}$ respectively did not differ significantly when pan-broiled to $60{ }^{\circ} \mathrm{C}$.

Pan material. To investigate the effect of pan material on cooking time, we tested cast aluminum, cast iron, stainless steel, and two composite pans. The composite pans had a copper and aluminum core respectively, and had a $1 \mathrm{~mm}$ layer of stainless steel cladding on the top and bottom surface.

As copper has the highest thermal diffusivity, followed by aluminum, cast iron, and lastly, stainless steel, this theoretically means that steaks seared in copper pans would cook the fastest, with all other parameters kept constant. However, due to its reactivity with acidic food, copper pans are rarely sold without first being coated in another non-reactive metal, typically, stainless steel. As stainless steel conducts heat relatively poorly, the addition of the material may negate the effect of using a relatively expensive copper core.

Indeed, our results agreed with this hypothesis as the composite copper pan fared the worst, taking the longest to cook a steak compared to the other materials used. However, this finding may not be valid if thinner layers (less than $1 \mathrm{~mm}$ ) of stainless steel are used. ${ }^{3}$

\footnotetext{
${ }^{2}[19]$ reported that the composition of muscle is 75 percent water, 20 percent protein, 3 percent fat, and 2 percent soluble non-protein substances.

${ }^{3}$ See Appendix B for our results on pan material.
} 


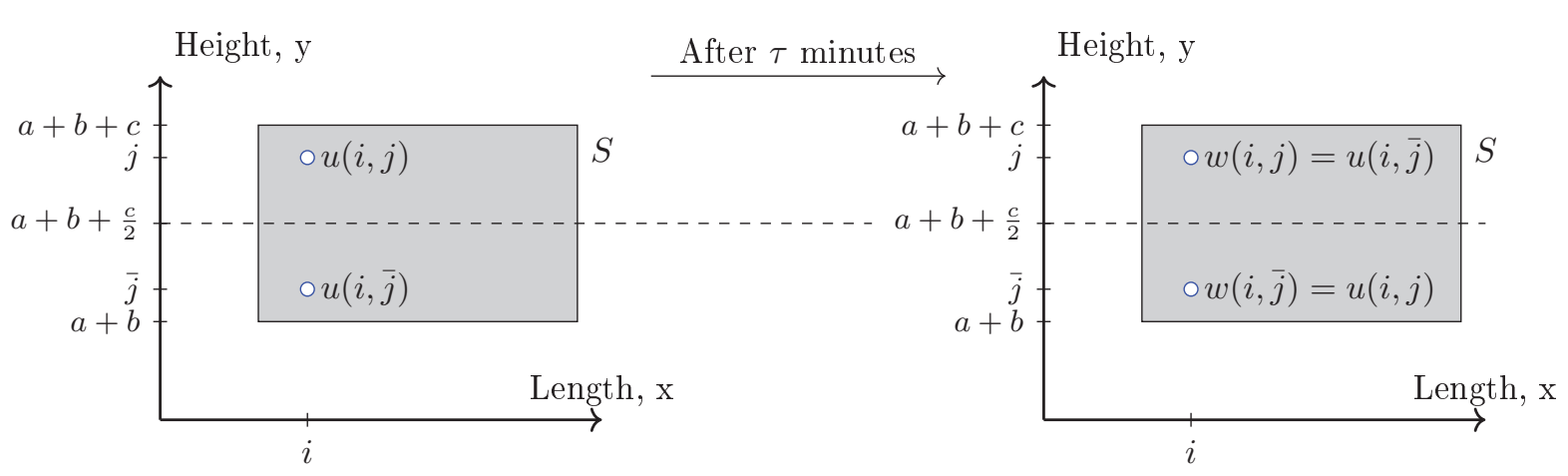

Figure 2.3: Illustration of the flipping function for the steak, $S$, at some $t$ and $t+\tau$ minutes.

After running a linear regression model for cooking time as a function of thermal diffusitivity and pan type, we found that the p-value was non-significant - the two variables cannot be used to predict cooking time. Therefore, we used a cast iron pan with the genetic algorithm in section 3 .

The heating process with time-based flipping criteria. We decided to heat the steak with a cast iron pan, flipping it every $2,4,6,8$, and 10 minutes, and observe the effect on cooking time and the temperature of the steak.

After conducting these simulations, we found that flipping the steak too often, that is, every two to four minutes, results in the lowest steak temperature. On the other hand, waiting too long to flip the steak, more than 10 minutes for example, was also not ideal as one side would be under-cooked. Therefore, we decided to set the minimum interval between flips, $t_{f}$, to 30 seconds, 1 minute, and 2 minutes.

Maximum temperature at the base of the pan, $u_{f}$. We hypothesized that a lower $u_{f}$ would result in a lower mean historical temperature. However, if $u_{f}$ was too low, the core of the steak would likely not be able to achieve a minimum temperature of $50{ }^{\circ} \mathrm{C}$ after 20 minutes. Thus, we had to ensure that the $u_{f}$ chosen was realistic in that it was a temperature easily achieved by a gas stove in practice, but also high enough to adequately heat a steak.

The authors of $[3,15]$ found that butane-fueled domestic gas stoves heated pans to a maximum temperature of $180-200{ }^{\circ} \mathrm{C}$ when the knob of the stove was turned to medium heat. In both papers, a heat sink was provided so that the pan was not able to reach an equilibrium temperature. Conversely, the authors of [10] found that domestic gas stoves powered by methane gas could heat a metal plate to a maximum temperature of $900{ }^{\circ} \mathrm{C}$ and a minimum of $200{ }^{\circ} \mathrm{C}$.

However, as portable cooking gas tends to be a mixture of butane and propane while piped gas tends to be methane, which is relatively less common in Asia, we decided to set $u_{f}$ to $170{ }^{\circ} \mathrm{C}$.

2.7. Flipping the steak. To simulate the flipping of a steak, we implemented a function that would swap the temperature of all the points in $S$ along the axis $y=a+b+\frac{c}{2}$.

Then, let $u(i, j)$ be the temperature at point $(i, j)$ before the flip, at $t=t_{f}$, and $w(i, j)$ be the temperature after, at $t=t_{f}+\tau$, as seen in Figure 2.3, where $\tau$ is the time-step. 


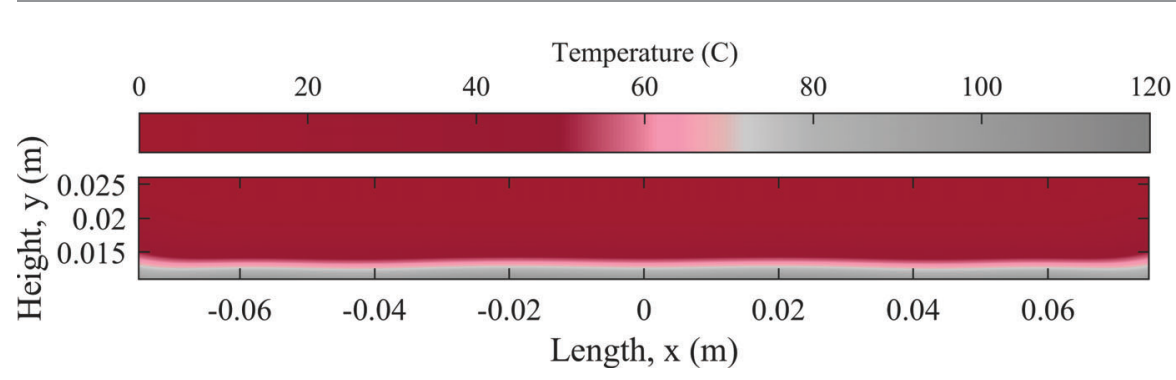

(a) Steak temperature after 2 mins of heating, before flipping.

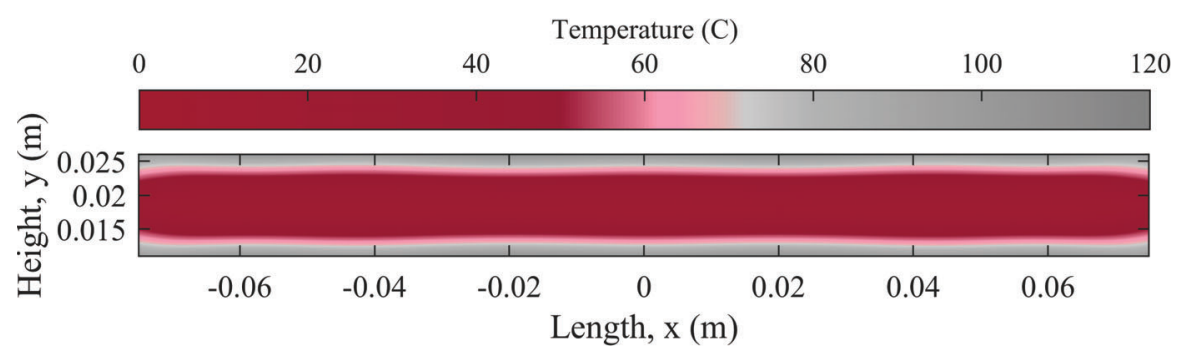

(c) Steak temperature after flipping and an additional 1 min of heating (total heating time of $3 \mathrm{~min}$ ).

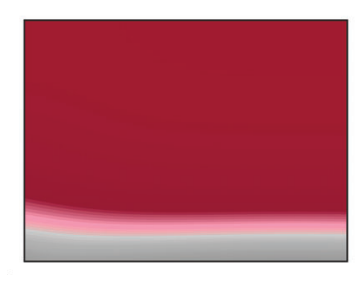

(b) Detail of Figure 2.4a.

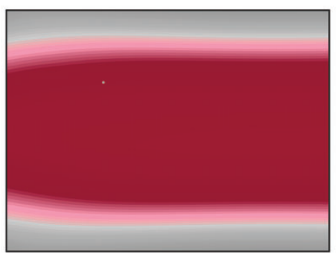

(d) Detail of Figure 2.4c.

Figure 2.4: A graphical representation of the heating process before and after flipping.

Then, given the temperature before the flip, $u$, at the point $(i, \bar{j})$, where $\bar{j}=a+b+(a+$ $b+c-j)$, we can determine the temperature after the flip, $w$, at $(i, j)$ with:

$$
F:=w(i, j)=u(i, \bar{j}), \quad(i, j),(i, \bar{j}) \in S, t=t_{f}+\tau .
$$

To see how the temperature at various points in the model change before and after a flip, see Figures $2.4 \mathrm{a}$ to $2.4 \mathrm{~d}$.

3. Optimization of the heating process with a genetic algorithm. As we wanted a medium-rare steak without raw spots, the steak had to be heated to at least $50{ }^{\circ} \mathrm{C}$. To ensure that the steak does not dry out, the overall mean temperature must be minimized, where a mean of about $60{ }^{\circ} \mathrm{C}$ indicates a high quality steak.

To do this, we first modified the flipping function from subsection 2.7 to allow the steak to be flipped at most once every $t_{f}$ seconds. As the partial differential equation described in subsection 2.1 was now highly discontinuous and non-linear, we decided to use a genetic algorithm to find a string of 0 s and 1 s that produced the lowest mean temperature, subject to a minimum temperature to ensure that the core of the steak did not remain cold. Here, 0 denoted that the steak would not be flipped while 1 denoted that the steak would be flipped,

For example, if $t_{f}=2 \mathrm{~min}$, then the solution vector $\boldsymbol{v}=$ [10011] would tell our program to flip the steak at the two and eight minute mark of the heating process, and to terminate the process after 10 minutes.

As the genetic algorithm requires us to fix the maximum number of entries in the solution vector, we had to specify a maximum cooking time before beginning the algorithm. We set this 
maximum to 20 minutes and divided it by $t_{f}$ to obtain the number of entries in the solution vector.

More formally, the flipping function would be performed when the $i^{\text {th }}$ entry in $\boldsymbol{v}$ is 1 and when

$$
t=i \cdot t_{f}+\tau, \quad \text { for } i=1,2, \ldots, \frac{20}{t_{f}}-1,
$$

while the finite element method would be carried out for all other values of $t$.

Additionally, we define the maximum historical temperature, $u_{h}$, on $S$ to be the maximum temperature at that same point during the entire heating process, which can be written as

$$
u_{h}(x, y):=\max _{0<t<20} u(x, y), \quad(x, y) \in S
$$

The maximum historical temperature, $u_{h}$, is used instead of the temperature at the end of heating process as the temperature of the steak oscillates depending on the side that is in contact with the pan.

Then, the genetic algorithm is used to minimize the following problem:

$$
\begin{array}{ll}
\min _{\boldsymbol{v} \in\{0,1\}^{n}} \operatorname{mean}\left(u_{h}(x, y ; \boldsymbol{v})\right), \quad(x, y) \in S \\
\text { s.t. } & u_{h}(x, y ; \boldsymbol{v}) \geq 50 \quad \forall(x, y) \in S \\
& \left.u(x, y, t)\right|_{t=i \cdot t_{f}+\tau}= \begin{cases}F(x, y), & \text { if } v_{i}=1, \quad(x, y) \in S, \\
\left.u(x, y, t)\right|_{t=i \cdot t_{f}}, & \text { if } v_{i}=0, \quad(x, y) \in S, \\
\left.u(x, y, t)\right|_{t=i \cdot t_{f}}, & \text { if }(x, y) \in P \cup O,\end{cases} \\
& \left.u(x, y, t)\right|_{t \neq i \cdot t_{f}+\tau} \text { is governed by the model in subsections } 2.1 \text { to } 2.5
\end{array}
$$

where $v_{i}$ is the $i^{\text {th }}$ entry of the solution vector $\boldsymbol{v}, u$ is the temperature in the specified domains, $F$ is the flipping function defined in subsection 2.7, and $\tau$ is the time step.

Additionally, given a maximum cooking time of 20 minutes, we want to be able to stop the heating procedure at any time to obtain a lower mean $u_{h}$. Therefore, we further modified our fitness function to find and stop at the last entry that was equal to 1, then output the mean $u_{h}$.

Then, we can update section 3 to:

$$
\left.u(x, y, t)\right|_{t=i \cdot t_{f}+\tau}= \begin{cases}F(x, y), & \text { if } v_{i}=1 ; \forall j>i, v_{j} \text { are not all } 0 ; \quad(x, y) \in S, \\ \left.u(x, y, t)\right|_{t=i \cdot t_{f}}, & \text { if } v_{i}=1 ; \forall j>i, v_{j}=0 ; \quad(x, y) \in S, \\ \left.u(x, y, t)\right|_{t=i \cdot t_{f}}, & \text { if } v_{i}=0, \quad(x, y) \in S, \\ \left.u(x, y, t)\right|_{t=i \cdot t_{f}}, & \text { if }(x, y) \in P \cup O .\end{cases}
$$

For example, if the solution vector was $\boldsymbol{v}=\left[\begin{array}{llll}1 & 0 & 1 & 0\end{array}\right]$, then the heating process would stop after $3 t_{f}$ minutes, despite having a maximum heating time of $5 t_{f}$ minutes.

The genetic algorithm solver used in this paper consists of the following components: an initial population (or the first generation), a fitness function, a method of creating the next generation, and a stopping criteria. The following sections explain the process of the genetic algorithm and note the parameters used. For a flowchart of these procedures, see Figure 3.1. 


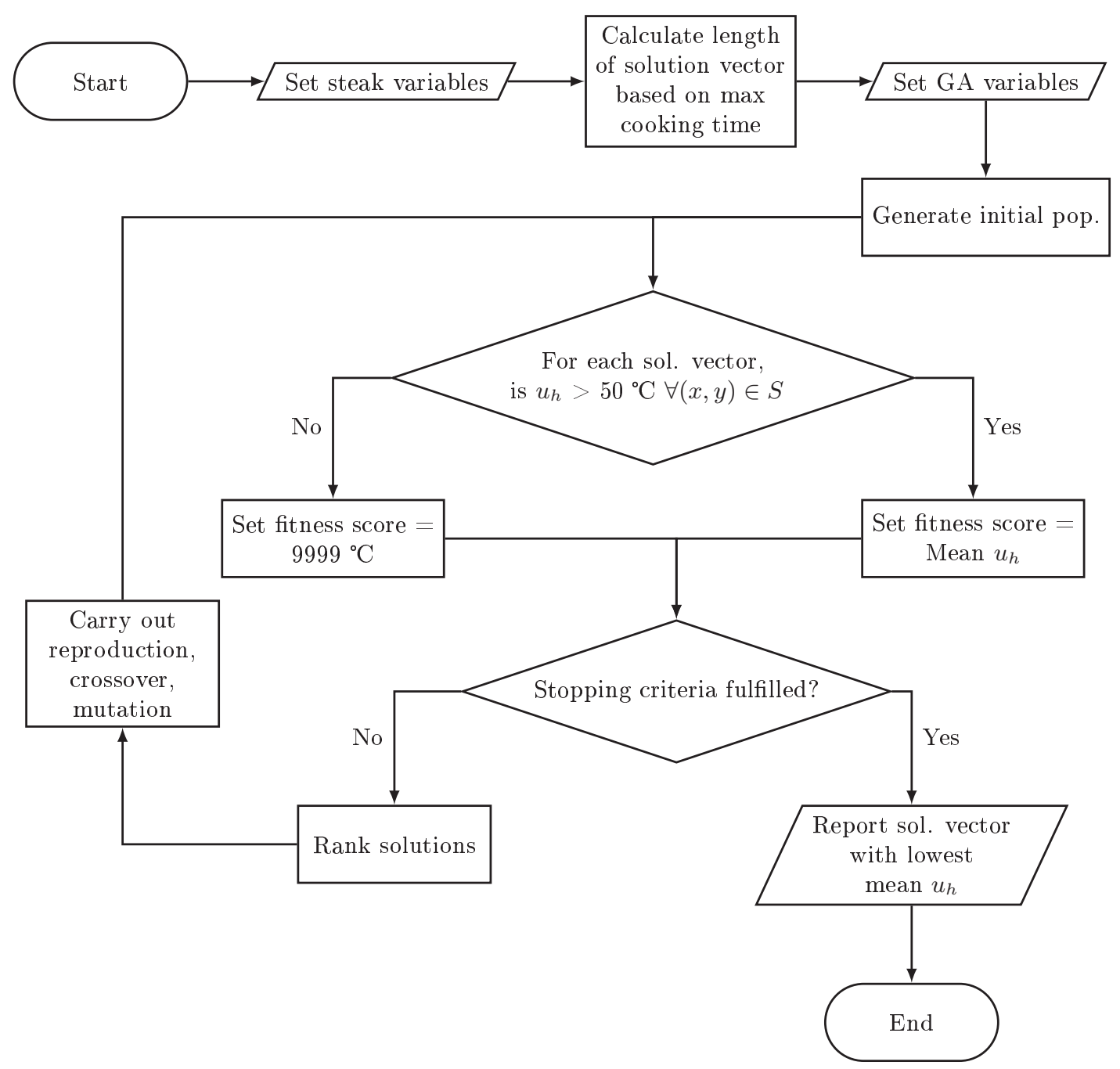

Figure 3.1: Computational procedure for the modeling and optimization process.

3.1. Initial population. An initial population consists of solutions to the problem posed which we set to produce a random, uniformly distributed, binary initial population.

3.2. Fitness function. An important component of the genetic algorithm is the fitness function which determines if a solution $\boldsymbol{v}$ should be carried over to the next generation.

As we wanted a medium-rare steak where the steak is heated to between 55 and $65{ }^{\circ} \mathrm{C}$, we set the fitness function to output the mean maximum historical temperature $u_{h}$ of the steak. A lower mean $u_{h}$ indicates higher fitness, and would give the solution higher priority when generating the subsequent generation. Thus, the fitness function was to be minimized by the genetic algorithm.

To ensure that the steak is adequately cooked, we specified that $u_{h}$ at each node must be at least $50{ }^{\circ} \mathrm{C}$. Otherwise, the solution would be assigned a dummy value of $9999{ }^{\circ} \mathrm{C}$ to denote poor fitness. 
3.3. Subsequent populations. The genetic algorithm produce a new generation of solutions with a selection, crossover, and mutation function.

Selection function. Before solution vectors were selected for crossover, the solutions were first ranked according to their fitness score, where a lower score is better. Then, this fitness was scaled by the function (Fitness value $/ \sqrt{r}$ ) where $r$ is the rank of the solution [17]. Lastly, a selection function picked two parents based on their scaled fitness value.

Crossover function. To simulate the evolutionary process of organisms in a population, the genetic algorithm consists of a crossover function that selects two parents, as determined by the selection function, and then creates a child (or a new solution) by selecting several entries from both parents and combining them [17].

Here, we used a crossover function that sets the $i^{\text {th }}$-entry of the child's solution equal to either the $i^{\text {th }}$-entry from Parent $\mathrm{A}$ or the $i^{t h}$-entry from Parent B with equal probability. A maximum of 80 percent of the child's entries undergo crossover while the remaining entries are copied from the fitter parent [17].

To ensure that the best solution from each population is preserved, we specified that this solution be cloned so that it survives, unchanged, to the next generation.

Mutation function. To introduce new solutions into the population, a uniformly distributed mutation function was used to modify the entries of the solution vectors. Each entry had a 5 percent probability of being mutated, or being swapped for a 0 or 1 , depending on its original state.

3.4. Stopping criteria. As solutions obtained by the genetic algorithm are unlikely to be unique, or the "best" solution, we set several stopping criteria to ensure that the already time-consuming process did not continue indefinitely.

We set the maximum number of generations to 20 , and set the maximum stall generation to five with a tolerance of 1 . This meant that if the mean $u_{h}$ did not decrease by at least $1{ }^{\circ} \mathrm{C}$ for five consecutive generations, the genetic algorithm is to quit and output $u_{h}$.

As the GA would likely produce a different solution each time, we repeated the procedure two more times and recorded the best solution of the three.

4. Model evaluation. In this section, we explain how the finite element method was set up and used to compute the temperature of a steak at different times. Then, we compare the model derived to empirical results.

4.1. Numerical simulations. The domain was discretized in space by generating a mesh for the domain with the distance between nodes set to $5 \cdot 10^{-4} \mathrm{~m}$. There was no significant improvement when the spatial step-size was reduced to $1 \cdot 10^{-4} \mathrm{~m}$. The domain was also discretized in time, with the time-step set to 0.5 seconds, and the model was then simulated for a total of 20 minutes using the finite-element method. In particular, a transient, general PDE model was used, as opposed to a thermal or structural model.

The flipping function in subsection 2.7 was implemented by stopping the simulation when the steak fulfills a predetermined criteria, and restarting the simulation with initial conditions as in (2.25). This function fetched temperature data at node points with an interpolation function. 


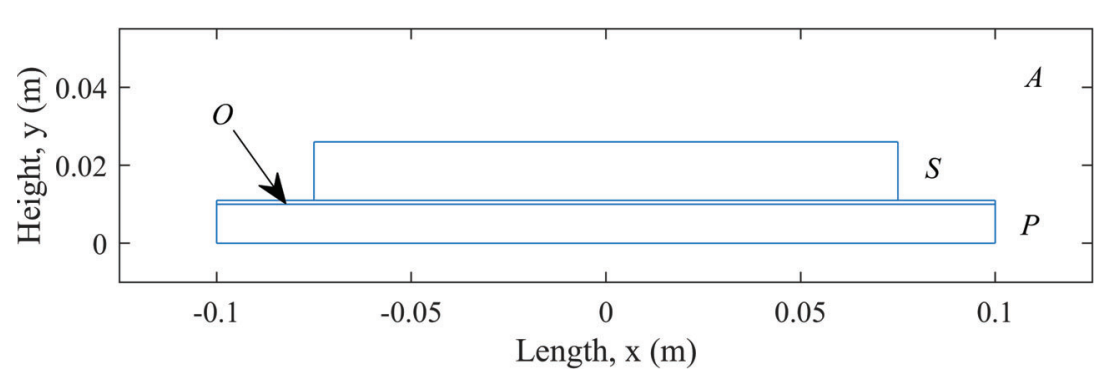

(a) Cross-sectional view of the modified model.

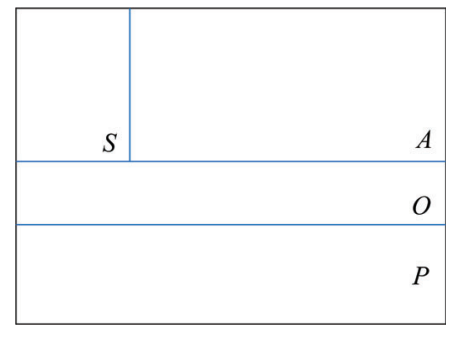

(b) Detail of oil layer.

Figure 4.1: The modified model used for verification.

If the steak was turned over based on the temperature at the midpoint depth, such as in subsection 4.2, we calculated the average temperature at a 151 linearly-spaced points, $u_{\text {mid }}$, every second until $u_{m i d}$ is greater or equal to the specified temperature before flipping the steak. Otherwise, the scheme set out in (3.7) was used. Note that $u_{m i d}$ was found with an interpolation function as well.

4.2. Evaluation results. First, we assumed that the steaks used in $[16,7]$ were lean, and had a radius of $0.075 \mathrm{~m}$. Then, as [16, 7] both used cast aluminum pans but did not note the type of alloy used, we chose an alloy with 84 percent aluminum.

In [7], the steaks were flipped when the core reached $42{ }^{\circ} \mathrm{C}$, while the steaks in [16] were flipped after 4 and 6 minutes regardless of their temperature. Therefore, we modified the simulation to account for the different procedures.

Both $[16,7]$ pan-broiled their steaks, that is, they used a minimal quantity of oil, or no oil at all. Therefore, we reduced the oil in the model from having a depth of $2 \mathrm{~mm}$ to a depth of $1 \mathrm{~mm}$, thus producing a second, modified model. However, the PDE and boundary conditions remained the same. See Figure 4.1 for a graphical representation of the modified model.

The modified model was discretized and simulated as specified in subsection 4.1. Table 4.1 summarizes the results obtained in the simulations and compares it to the empirical results.

From the results, we observed that the cooking times predicted by our model is, with the exception of the first case, within the range of experimental values. Therefore, we claim that the model is accurate.

5. Results and discussion. Table 5.1 summarizes the results obtained from both the controlled simulation, where the steaks were only flipped once, halfway through the cooking procedure, and the solution obtained from the genetic algorithm, where the steak was allowed to be flipped at most once every $t_{f}$. The steak was modeled on a $500 \mathrm{~g}, 2.5 \mathrm{~cm}$ thick rib-eye steak, with all simulations conducted in MATLAB [18].

From Table 5.1, it is clear that the solution vectors obtained are not the "best" solutions. For example, the solution when $t_{f}=0.5$ min yields a mean $u_{h}$ that is higher than when $t_{f}=$ 1 min. However, the latter solution vector can be easily transformed into a solution vector for $t_{f}=0.5 \mathrm{~min}$ - by adding a zero after every entry. Then, we would have obtained a mean $u_{h}$ of $69{ }^{\circ} \mathrm{C}$ for $t_{f}=0.5 \mathrm{~min}$, which is objectively better than a mean of $72{ }^{\circ} \mathrm{C}$.

Despite this, the steaks produced with the genetic algorithm are far superior to the ones 
Table 4.1: Comparison of total cooking time for experimental and simulated processes. Total cooking time was recorded when mean temperature at the midpoint depth reaches the target temperature.

\begin{tabular}{|c|c|c|c|c|c|}
\hline \multirow{2}{*}{$\begin{array}{l}\text { Height of } \\
\text { steak, cm }\end{array}$} & \multicolumn{2}{|c|}{ Flip time, $t_{f}, \min$} & \multirow{2}{*}{$\begin{array}{l}\text { Target } \\
\text { temp, }{ }^{\circ} \mathrm{C}\end{array}$} & \multicolumn{2}{|c|}{ Time to cook, $\min$} \\
\hline & Experiment & Simulation & & Experiment & Simulation \\
\hline \multicolumn{6}{|l|}{ From [16] } \\
\hline 1.5 & 7 & 5.8 & 65 & $16.3 \pm 2.0$ & 13.5 \\
\hline 2.5 & 20 & 13.5 & 65 & $27.2 \pm 5.4$ & 30.0 \\
\hline \multicolumn{6}{|l|}{ From [Y] } \\
\hline \multirow[t]{4}{*}{2.0} & \multirow[t]{4}{*}{4} & \multirow[t]{4}{*}{$\mathrm{n} / \mathrm{a}$} & 60 & $15.5 \pm 2.64$ & 12.0 \\
\hline & & & 63 & $11.9 \pm 2.02$ & 13.0 \\
\hline & & & 67 & $15.5 \pm 2.80$ & 14.5 \\
\hline & & & 71 & $18.5 \pm 2.64$ & 16.4 \\
\hline \multirow[t]{4}{*}{2.0} & \multirow[t]{4}{*}{6} & \multirow[t]{4}{*}{$\mathrm{n} / \mathrm{a}$} & 60 & $12.0 \pm 1.05$ & 12.9 \\
\hline & & & 63 & $13.0 \pm 1.63$ & 14.0 \\
\hline & & & 67 & $16.4 \pm 1.71$ & 15.6 \\
\hline & & & 71 & $18.6 \pm 2.55$ & 17.7 \\
\hline
\end{tabular}

Table 5.1: Mean maximum historical temperature, $u_{h}$, of the steaks with one flip only or multiple flips as found by the genetic algorithm.

\begin{tabular}{|c|c|c|c|c|}
\hline$t_{f}, \min$ & Solution Vector, $\boldsymbol{v}$ & Total time, $\min$ & Min $u_{h},{ }^{\circ} \mathrm{C}$ & Mean $u_{h},{ }^{\circ} \mathrm{C}$ \\
\hline \multicolumn{5}{|c|}{ Control (Single flip) } \\
\hline $\mathrm{n} / \mathrm{a}$ & Flip once, at 9 minutes & 19 & 53 & 82 \\
\hline $\mathrm{n} / \mathrm{a}$ & Flip once, at 9 minutes & 18 & 52 & 81 \\
\hline $\mathrm{n} / \mathrm{a}$ & Flip once, at 8 minutes & 16 & 50 & 80 \\
\hline \multicolumn{5}{|c|}{ With genetic algorithm (Multiple flips) } \\
\hline 0.5 & $\begin{array}{c}{[10010000000010101001} \\
01110001000011110110]\end{array}$ & 19.5 & 50 & 72 \\
\hline 1 & [01011010110101110110] & 19 & 50 & 69 \\
\hline 2 & {$[1111011110]$} & 18 & 50 & 74 \\
\hline
\end{tabular}

in the controlled simulation, even when the cooking time of the controlled simulation was shortened to 16 minutes. (Note that the total cooking time could not be further reduced while maintaining a minimum $u_{h}$ of $50{ }^{\circ} \mathrm{C}$ ). When comparing the control steaks to the GA steaks, we observe that the former have much higher temperatures than the latter, with a difference of about $10{ }^{\circ} \mathrm{C}$. As we also aimed to produce steaks with a uniform temperature gradient, we notice that the control steaks in Figures 5.1a to 5.1c do not meet this goal, showing high variation in temperature along the vertical axes.

From the graphical representation of the GA steaks in Figures 5.1d to 5.1f, we see that the steak in Figure 5.1e fared the best, having the least amount of overcooked gray meat. This is important as one method of determining the doneness of a steak is by observing its color. In particular, a medium-rare steak is said to have a 50 percent red core with minimal graying. Coincidentally, the steak in Figure 5.1e also had the lowest mean $u_{h}$. We also observe that 


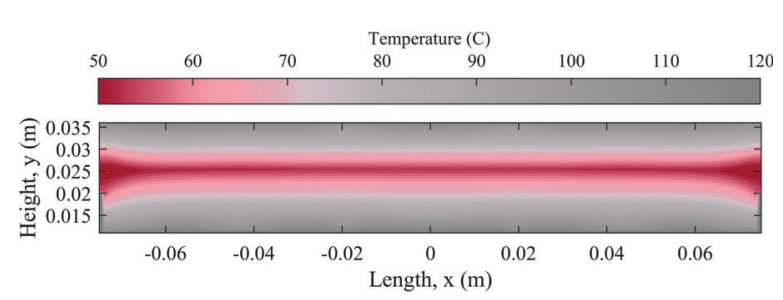

(a) Control 1: 19 minutes of heating

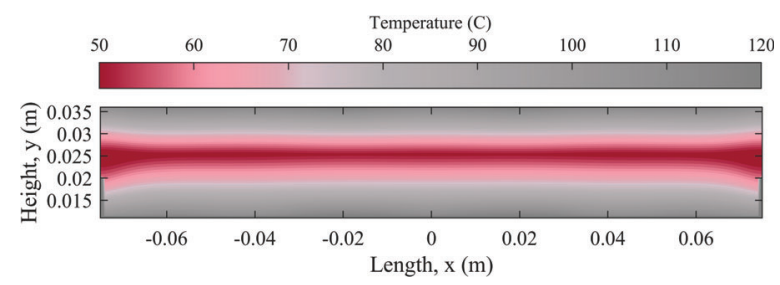

(c) Control 3: 16 minutes of heating

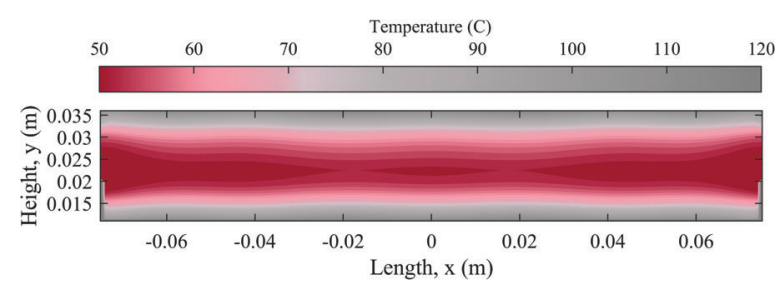

(e) GA 2: $t_{f}=1 \mathrm{~min}$.

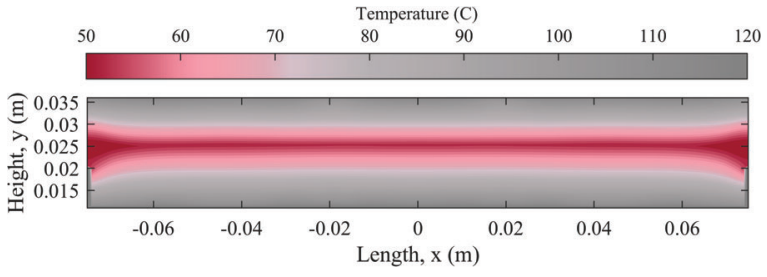

(b) Control 2: 18 minutes of heating

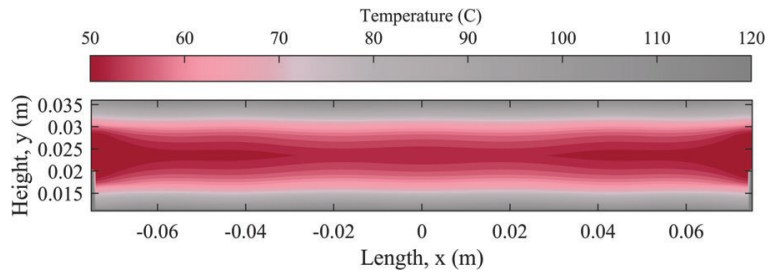

(d) GA 1: $t_{f}=0.5 \mathrm{~min}$

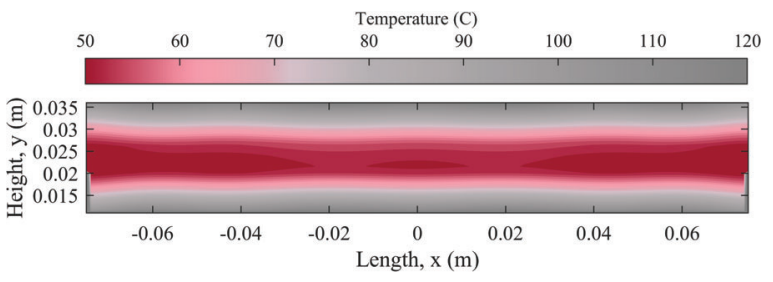

(f) GA $3: t_{f}=2 \min$.

Figure 5.1: Maximum historical temperature, $u_{h}$, of control and GA group, with various heating times and $t_{f}$ respectively.

Table 5.2: Mean maximum historical temperature, $u_{h}$, of the steaks when flipped at most once every $t_{f}$ minutes; found with the genetic algorithm with fixed physical parameters, and preliminary results of GA with other parameters.

\begin{tabular}{|l|c|c|c|c|c|c|}
\hline$t_{f}$, min & $\begin{array}{c}\text { Height of } \\
\text { steak, cm }\end{array}$ & $\begin{array}{c}\text { Temp of heat } \\
\text { source, }{ }^{\circ} \mathrm{C}\end{array}$ & $\begin{array}{c}\text { Steak initial } \\
\text { temp, }{ }^{\circ} \mathrm{C}\end{array}$ & $\begin{array}{c}\text { Room temp, } \\
{ }^{\circ} \mathrm{C}\end{array}$ & $\begin{array}{c}\text { Total time } \\
\text { cooked, min }\end{array}$ & $\begin{array}{c}\text { Mean } u_{h}, \\
{ }^{\circ} \mathrm{C}\end{array}$ \\
\hline Results from Table 5.1 & \multicolumn{5}{l}{} \\
\hline $0.5 / 1 / 2$ & 2.5 & 170 & 20 & 20 & $19.5 / 19 / 18$ & $72 / 69 / 74$ \\
\hline \multicolumn{7}{|l|}{ Preliminary results with other parameters } \\
\hline 1 & 2 & 140 & -2 & 22 & 19 & 64 \\
\hline 1 & 3 & 220 & -2 & 25 & 19 & 72 \\
\hline
\end{tabular}

the historical temperature of the steaks follow a wave-like pattern, which is likely the effect of using a Gaussian function to simulate a gas burner.

Therefore, based on the simulation results, we conclude that flipping the steak once every 1 to 2 minutes results in a more evenly-cooked steak compared to flipping the steak only once.

Additionally, we briefly tested the GA on steaks with different dimensions, initial temperatures, heat sources, and room temperatures. As seen in Figure 5.2, these steaks were cooked 


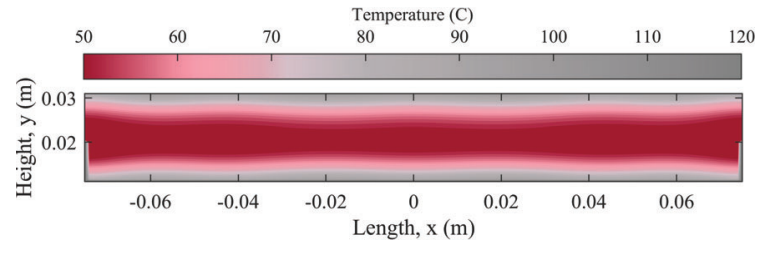

(a) $2 \mathrm{~cm}$-thick steak with room temperature of $22{ }^{\circ} \mathrm{C}$, heat source of $140{ }^{\circ} \mathrm{C}$.

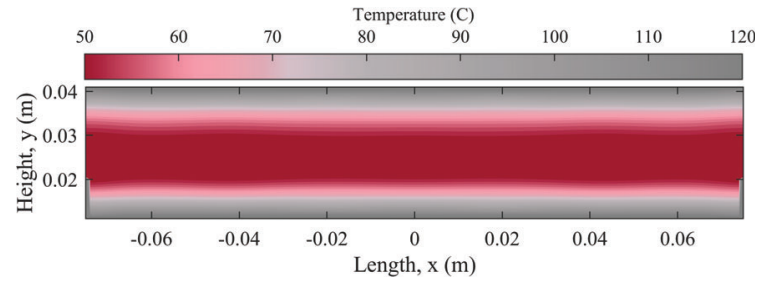

(b) $3 \mathrm{~cm}$-thick steak with room temperature of $25{ }^{\circ} \mathrm{C}$, heat source of $220{ }^{\circ} \mathrm{C}$.

Figure 5.2: Maximum historical temperature of steaks found with GA given different physical parameters.

reasonably well with minimal over-cooking and mean $u_{h}$ of 64 and $72{ }^{\circ} \mathrm{C}$ respectively. See Table 5.2 for a summary of these preliminary results.

A caveat to note with our findings is that the internal temperature of the GA steaks were $50{ }^{\circ} \mathrm{C}$, well below the $63{ }^{\circ} \mathrm{C}$ minimum recommended by the USDA for beefsteaks [7]. However, without too much work, the GA can be repeated for different target minimum temperatures, for example, to cook a steak to medium or well-done. The GA developed in this paper can also be modified to find the optimum temperature of the heat source at different times, as done in [2], or the optimum total cooking time, or some combination of the two. The ranking function used by the GA can also be further improved upon in future work in order to differentiate between solution vectors with the same fitness score. For example, the ranking function used could prioritize solution vectors with less flips over those with more flips if cooking procedures with less flips are preferred.

The simulation procedure was conducted with a four-core Intel i7 processor with each GA simulation taking around 20 hours to complete. However, the results from GA were unverified as it was beyond the scope of this paper. To reduce computation time, we suggest that the GA procedure be done on a cloud server or high-performance cluster, or a faster stochastic method be utilized, such as the differential evolution used in [2], or another method, such as pattern search, be applied in combination with GA.

To verify the results found with GA, we recommend that the GA described in this paper be run multiple times with different input variables, such as room temperature and steak dimensions, to obtain a number of solutions. These solutions can then be used in a regression model to predict flip-times given the different inputs. Finally, a similar empirical model can be prepared in a laboratory setting for verification.

Furthermore, the model is limited by the many assumptions and approximations used to determine mass transfer as seen in Appendix C. Therefore, a more complex model that considers the porosity of meat, as demonstrated in [4], and allows for shrinkage, as presented in [13], can be simulated and optimized with GA. The exclusion of shrinkage from the model used in this paper is likely the main cause for the uneven heating along the vertical edges, as seen in Figures 5.1a to 5.1f, as we would expect that these edges, having been in contact with hot oil, to be at least the same temperature as the horizontal edges. Moreover, we recommend that future work, if shrinkage and a more sophisticated mass transfer model is used, set the thermaldiffusivityof thesteakas a variabledependenton historicaltemperature 
as the composition and elasticity of meat changes when heated. However, further work can be done here to investigate the utility of using a variable thermal diffusivity as the model, as our model was found to be well within acceptable ranges despite using an estimated, constant thermal diffusivity.

Additionally, although we found that pan material does not have a significant effect on cooking time, as noted in subsection 2.6, our model was limited to single-material pans and composite pans with thin stainless steel cladding. Therefore, we are unsure if our findings apply to pans with more elaborate construction and coatings, and recommend that further work be done here.

Finally, as we assumed that the model was radially symmetrical to reduce its dimensions from three to two, future work can derive and solve a three-dimensional model, at the cost of longer computational time.

6. Conclusions. Using a parabolic partial differential equation and boundary conditions that simulate diffusion, convection, and a heat source, we derived a model that simulates the heating of a steak reasonably well when compared to empirical data. We also investigated and estimated various physical parameters that may affect cooking time such as room temperature, steak initial temperature, and heat source temperature. Lastly, we found that using a stochastic approach to determine when a flip should take place, with small time intervals between each flip, is superior to a single-flip halfway through the cooking process as it minimizes over-cooking. However, the method used, the genetic algorithm, is computationally expensive and may not be practical for more complex two- and three-dimensional models. The model derived in this paper also assumes a fixed domain and constant thermal diffusivity, and relies on a simplified mass transfer process. Further work can be done to improve the mathematical model used and to empirically verify the results found with GA.

Acknowledgements. I would like to thank my adviser, Prof. Leevan Ling of Hong Kong Baptist University, without whom I would never have been able to complete this project, let alone begin it. He recommended my initial project to the Math Department of HKBU for a summer REU, gave me pointers on where and what to research, helped me refine my research topic, and pointed out areas in my research that needed to be improved upon. He suggested I look into the genetic algorithm to find an optimum solution for my model and painstakingly explained how it worked. Finally, he provided many ideas of how this project could be applied and improved upon in the future.

I would also like to thank Prof. Xue-Cheng Tai for observing my oral presentation and providing feedback on areas I could improve on.

Finally, I would like to express my gratitude to the two reviewers whose comments and suggestions were an integral part of improving this manuscript.

\section{REFERENCES}

[1] J. M. Adler, I. Geornaras, K. E. Belk, G. C. Smith, and J. N. Sofos, Thermal inactivation of escherichia coli o15\%:h7 inoculated at different depths of non-intact blade-tenderized beef steaks, J. Food Sci., 77 (2012), pp. 108-114, https://doi.org/10.1111/j.1750-3841.2011.02552.x.

[2] J. Banga, Z. Pan, And R. Singh, On the optimal control of contact-cooking processes, Food Bioprod. Process., 79 (2001), p. 145-151, https://doi.org/10.1205/096030801750425235. 
[3] J. Cernela, B. Heyd, And B. Broyart, Evaluation of heating performances and associated variability of domestic cooking appliances (oven-baking and pan-frying), Appl. Therm. Eng., 62 (2014), pp. 758765, https://doi.org/10.1016/j.applthermaleng.2013.08.045.

[4] M. Chapwanya and N. N. Misra, A mathematical model of meat cooking based on polymer-solvent analogy, Appl. Math. Model., 39 (2015), p. 4033-4043, https://doi.org/10.1016/j.apm.2014.12.015.

[5] J. F. EBerth, J. A. NeAl, And F. C. R. Hernandez, Evaluation of heat propagation through poultry in a reduced computational-cost model of contact cooking: Evaluation of heat propagation through poultry, Int. J. Food Sci. Technol., 47 (2012), p. 1130-1137, https://doi.org/10.1111/j.1365-2621.2012.02951.x.

[6] Engineering ToolBox, Air - dynamic and kinematic viscosity, 2003, https://www.engineeringtoolbox. com/air-absolute-kinematic-viscosity-d 601.html (accessed 2020/07/10).

[7] C. O. Gill, J. Devos, M. K. Youssef, And X. YAng, Effects of selected cooking procedures on the survival of escherichia coli o157:h7 in inoculated steaks cooked on a hot plate or gas barbecue grill, J. Food Prot., 77 (2014), pp. 919-26, https://doi.org/10.4315/0362-028X.JFP-13-526.

[8] D. W. Hahn and M. N. Ozisik, Heat Conduction, John Wiley \& Sons, Hoboken, New Jersey, 2012.

[9] J. P. Holman, Heat Transfer, McGraw-Hill, New York, New York, 2010.

[10] S.-S. Hou AND Y.-C. Ko, Effects of heating height on flame appearance, temperature field and efficiency of an impinging laminar jet flame used in domestic gas stoves, Energy Convers. Manage., 45 (2004), pp. $1583-1595$, https://doi.org/10.1016/j.enconman.2003.09.016.

[11] J. Inediala, L. Correia, G. Fenton, and N. Ben-Abdallah, Finite element modeling of heat transfer in meat patties during single-sided pan-frying, J. Food Sci., 61 (1996), p. 796-802, https: //doi.org/10.1111/j.1365-2621.1996.tb12205.x.

[12] M. Mitchell, An introduction to genetic algorithms, Complex adaptive systems, MIT Press, 1996.

[13] H. Nelson, S. Deyo, S. Granzier-Nakajima, P. Puente, K. Tully, and J. Webb, A mathematical model for meat cooking, Eur. Phys. J. Plus, 135 (2020), p. 322, https://doi.org/10.1140/epjp/ s13360-020-00311-0.

[14] D. Ou AND G. Mittal, Modeling and simulation of unfrozen hamburger single-sided panfrying with flippings for microbial safety, J. Muscle Foods, 17 (2006), p. 115-140, https://doi.org/10.1111/j. 1745-4573.2006.00031.x.

[15] F. Sanz-Serrano, C. Sagues, and S. Llorente, Inverse modeling of pan heating in domestic cookers, Appl. Therm. Eng., 92 (2016), pp. 137-148, https://doi.org/10.1016/j.applthermaleng.2015.09.084.

[16] C. Shen, J. M. Adler, I. Geornaras, K. E. Belk, G. C. Smith, And J. N. Sofos, Inactivation of escherichia coli o15\%:h7 in nonintact beefsteaks of different thicknesses cooked by pan broiling, double pan broiling, or roasting by using five types of cooking appliances., J. Food Prot., 73 (2010), pp. 461-469, https://doi.org/10.4315/0362-028X-73.3.461.

[17] The Mathworks, Inc., Genetic algorithm options, 2020, https://www.mathworks.com/help/gads/ genetic-algorithm-options.html (accessed 2020/11/23).

[18] The MathWorks, Inc., MATLAB, 2020, https://www.mathworks.com/products/matlab.html (accessed 2021/04/12). Version 2020a.

[19] E. Tornberg, Effects of heat on meat proteins - implications on structure and quality of meat products, Meat Sci., 70 (2005), pp. 493-508, https://doi.org/10.1016/j.meatsci.2004.11.021. 50th International Congress of Meat Science and Technology,(ICoMST), 8-13 August 2004, Helsinki, Finland.

[20] X. Yang, J. Devos, And M. D. Klassen, Inactivation of escherichia coli o157:h7 in minute steaks cooked under selected conditions, J. Food Prot., 80 (2017), pp. 1641-1647, https://doi.org/10.4315/ 0362-028X.JFP-17-081.

Appendix A. Derivation of the convection coefficient, $h$. In subsection 2.3, the derivation for the value of $h$ for a plane under free convection is not included. Below, we show that $h$ is a function of temperature, $u$, and that its calculation depends on the orientation of the plane.

For free convection, $h$ is defined as

$$
h=\frac{\mathrm{Nu}_{L} k}{L},
$$


Table A.1: These values are associated with free convection were taken from [9, p. 658] and [6].

\begin{tabular}{|l|l|c|c|}
\hline Symbol & Definition & Value & Units \\
\hline $\operatorname{Pr}$ & Prandtl number & 0.71 & nil. \\
\hline$k_{\text {air }}$ & Thermal conductivity of air & 1004 & $\mathrm{~W} / \mathrm{mK}$ \\
\hline $\mathrm{g}$ & Gravitational acceleration & 9.8 & $\mathrm{~m} / \mathrm{s}^{2}$ \\
\hline$\beta$ & Coefficient of thermal expansion of air & $3.32 \cdot 10^{-3}$ & $\mathrm{~K}^{-1}$ \\
\hline$\nu$ & Kinematic viscosity of air & $15.69 \cdot 10^{-6}$ & $\mathrm{~m}^{2} / \mathrm{s}$ \\
\hline
\end{tabular}

where $\mathrm{Nu}$ represents the Nusselt number, $k$ is the thermal conductivity of air, and $L$ is the characteristic length [9, p. 331].

In general, the Nusselt number is calculated as below:

$$
\mathrm{Nu}_{L}=C\left(\mathrm{Gr}_{L} \operatorname{Pr}\right)^{m}
$$

where Gr is the Grashof number, Pr is the Prandtl number, and $\mathrm{C}$, and $\mathrm{m}$ depended on the shape and configuration of the model [9, p. 332].

The Grashof and Prandtl number is defined as:

$$
\begin{aligned}
\operatorname{Gr}_{L} & =\frac{g \beta\left(u_{s}-u_{a t m}\right) L^{3}}{\nu^{2}} \\
\operatorname{Pr} & =\frac{\nu}{\alpha},
\end{aligned}
$$

where $g$ is gravitational acceleration, $\beta$ is the coefficient of thermal expansion of air, $\nu$ is the kinematic viscosity of air, and $\alpha$ is the thermal conductivity of air [9, p. 331].

As the Prandtl number for air is not affected by pressure or temperature, this value is assumed to be constant in our model [9, p. 658].

For horizontal planes, the Nusselt number and characteristic length, L, is determined as follows:

$$
\begin{aligned}
& \mathrm{Nu}_{L}= \begin{cases}0.54\left(\mathrm{Gr}_{L} \mathrm{Pr}\right)^{1 / 4} & \text { for } 2 \cdot 10^{4}<\mathrm{Gr}_{L} \operatorname{Pr}<8 \cdot 10^{6} \\
0.15\left(\mathrm{Gr}_{L} \mathrm{Pr}\right)^{1 / 3} & \text { for } 8 \cdot 10^{6}<\mathrm{Gr}_{L} \operatorname{Pr}<10^{11}\end{cases} \\
& L \quad=\frac{A}{p},
\end{aligned}
$$

where $A$ and $p$ is the area and perimeter of the surface respectively [9, p. 334].

For vertical planes, the following relation is used instead:

$$
\mathrm{Nu}_{L}=0.68+\frac{0.670 \mathrm{Ra}^{1 / 4}}{\left[1+(0.492 / \mathrm{Pr})^{9 / 16}\right]^{4 / 9}}, \quad \text { for } \mathrm{Ra}_{L}<10^{9}
$$

where $L$ is the height of the plane, $\mathrm{Ra}$ is the Rayleigh number, and is defined as the product of the Grashof number and Prandtl number [9, p. 334-335]. 


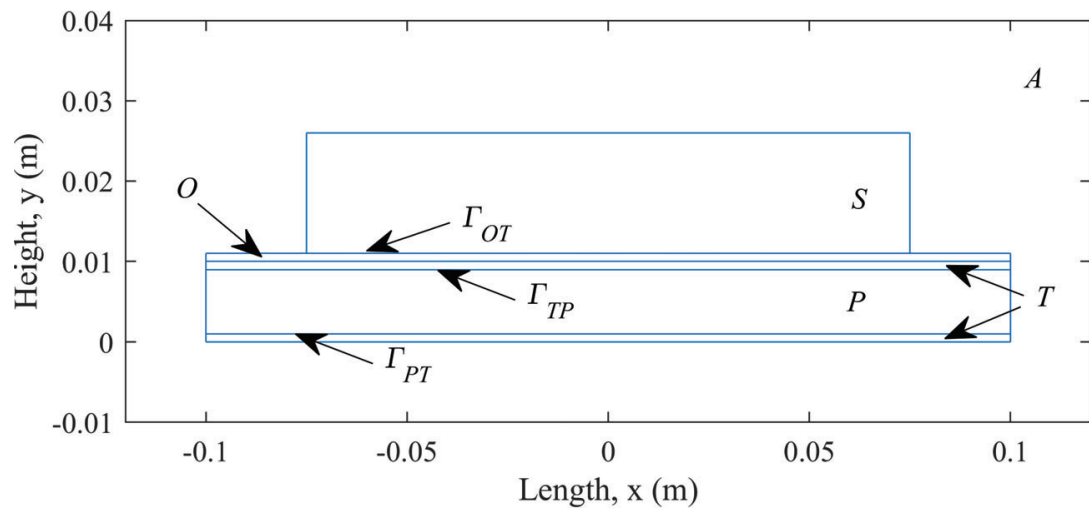

(a) Cross-sectional view of the modified model.

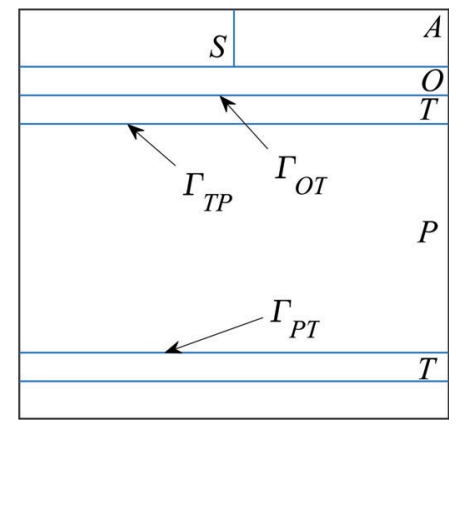

(b) Detail of pan layers.

Figure B.1: Cross-section of the modified model for testing different pan materials.

However, because the boundary condition would be discretized when solved, we set $L$ equal to the distance between nodes $h$ instead.

Appendix B. The heating process with different pan materials. In addition to the parameters listed in Table 2.1, the metals used were pure cast aluminum, cast iron, 18/8 stainless steel and copper 11000.

The composite aluminum-stainless steel and copper-stainless steel pans were $1 \mathrm{~cm}$ thick with a $8 \mathrm{~mm}$ layer of aluminum or copper sandwiched between two $1 \mathrm{~mm}$ layers of stainless steel. Here, the model was modified to include the new pan layers. See Figure B.1 for a graphical representation of this third model.

The partial differential equation used for the aluminum, cast iron, and stainless steel pans were as in section 2. However, the composite pans had an additional piece-wise partial differential equation and two additional boundary conditions governing the diffusion of heat between the layers:

$$
\begin{aligned}
k_{s t}\left[\frac{\delta^{2} u}{\delta x^{2}}+\frac{\delta^{2} u}{\delta y^{2}}\right] & =c_{s t} \rho_{s t} \frac{\delta u}{\delta t}, \quad(x, y) \in T, t>0 \\
\left.k_{p} \frac{\delta u}{\delta y}\right|_{y} & =\left.k_{o} \frac{\delta u}{\delta y}\right|_{y}, \quad(x, y) \in \Gamma_{T P} \cup \Gamma_{P T}, t>0,
\end{aligned}
$$

where $k_{s t}, c_{s t}$, and $\rho_{s t}$ was the thermal conductivity, specific heat, and density of stainless steel respectively, and $T$ was the 2-dimensional space occupied by the stainless steel cladding of the pan.

The flipping function was implemented when the average temperature at the midpoint depth of the steak reached $42{ }^{\circ} \mathrm{C}$, and deemed cooked when this value reached $65{ }^{\circ} \mathrm{C}$. Additionally, the maximum temperature at the top and bottom surface of the steak at the stopping time were recorded and summarized in Appendix B.

When using regression analysis to predict cooking time with the thermal diffusivity of the pan, and the type of pan, composite or normal, we found that these variables, thermaldiffusivity and pan tvpe.could not be used toredictcooking time ( $\mathrm{p}$-value $=0.3594$ ). 
Table B.1: Results of cooking time when using different pan materials.

\begin{tabular}{|l|c|c|l|l|}
\hline \multirow{2}{*}{ Type of pan } & \multirow{2}{*}{$\begin{array}{c}\text { Flipping } \\
\text { time, min }\end{array}$} & Cooking & \multicolumn{2}{|c|}{ Max temperature at } \\
\cline { 4 - 5 } & 5.82 & 13.42 & 112.68 & 127.81 \\
\hline Cast aluminum & 5.86 & 13.59 & 112.17 & 127.48 \\
\hline Cast iron & 6.01 & 14.18 & 110.69 & 126.54 \\
\hline Stainless steel & 5.99 & 14.15 & 110.88 & 126.24 \\
\hline Composite aluminum & 6.12 & 14.81 & 110.34 & 125.84 \\
\hline Composite copper & \multicolumn{3}{|l}{$\mathrm{C}$} & Bottom, \\
\hline
\end{tabular}

Appendix C. Derivation of heat flux, $Q$. Given the relationship $\Delta u=u_{w}-u_{\text {sat }}$, we assume that the temperature excess, $\Delta u$, does not exceed $100{ }^{\circ} \mathrm{C}$, and therefore, can use the equation for heat flux that corresponds to nucleate boiling, as it occurs when the excess temperature is between 1 and $100{ }^{\circ} \mathrm{C}$.

We also assume that net heat transfer is upwards, away from the pan, and can then use the following equations from $[9$, p. 507] to determine heat flux per unit area, $Q / A$ :

$$
\begin{cases}1000 \cdot 1042(\Delta u)^{1 / 3}, & \text { when } 0 \leq \Delta u \leq 7.76 \\ 1000 \cdot 5.56(\Delta u)^{3}, & \text { when } \Delta u>7.76,\end{cases}
$$

where $A$ is area and $u_{\text {sat }}$ is the boiling temperature of water at sea level.

As in subsection 2.6, we assumed again that the molecules of water and muscle are distributed randomly throughout the steak. Thus, we could take $u_{w}$ to be the temperature of the steak at the node, $u$.

As heat flux is calculated for every node set by the finite element method, we define $A=\pi\left(\frac{h}{2}\right)^{2}$, and multiply $Q / A$ by 0.75 to obtain the equation in subsection 2.4 . The equation is scaled by 0.75 to reflect the composition of the steak which is 75 percent water. 